\title{
Consensus Problem of Second-order Dynamic Agents with Heterogeneous Input and Communication Delays
}

\author{
C.-L. Liu, F. Liu
}

\author{
Cheng-Lin Liu, Fei Liu \\ Jiangnan University \\ Institute of Automation \\ Wuxi 214122, Jiangsu, People's Republic of China \\ E-mail: liucl@jiangnan.edu.cn, fliu@jiangnan.edu.cn
}

\begin{abstract}
Consensus problem of second-order multi-agent systems with velocity damping term in agent's dynamics is investigated. Based on frequency-domain analysis, decentralized consensus condition, which depends on the input delays, is obtained for the system based on undirected and symmetric graph with heterogeneous input delays. For the system based on directed graph with both heterogeneous input delays and communication delays, decentralized consensus condition, which is dependent on the input delays but independent on the communication delays, is also obtained. Simulations illustrate the correctness of the results.
\end{abstract}

Keywords: coordination control, consensus, second-order multi-agent systems, communication delay, input delay

\section{Introduction}

In the last decade, distributed coordination of multiple autonomous agents has attracted more and more attention from various research communities for its broad application including automated highway systems, air traffic control, congestion control in Internet, etc.

Consensus problem, which is one of the most fundamental and important issues in coordination control of multi-agent systems, requires that the outputs of several spatially distributed agents reach a common value without recurse to a central controller. For the first-order multi-agent systems with agents' dynamics modeled by single integrators and second-order multi-agent systems with agents' dynamics modeled by double integrators, consensus algorithms have been proposed to solve the consensus problem, and sufficient conditions have been obtained for the system converging to the consensus with static or switched interconnection topology [1-4].

Recently, more and more attention has been paid on the delay effect on consensus convergence of multi-agent systems. Generally speaking, two kinds of time delays cannot be negligible in the multiagent systems. One is communication delay, which is related to the information transmission between neighboring agents. The other is input delay, which is related to the processing and connecting time for the packets arriving at each agent [5].

Consensus problem under communication delays has been extensively studied for the first-order multi-agent systems based on different analysis methods, such as Lyapunov functions analysis [6,7], frequency-domain analysis [2,8], method based on delayed and hierarchical graphs $[9,10]$, method based on difference of maximum value and minimum value $[11,12]$, etc. However, consensus analysis of second-order multi-agent systems with communication delay is much more difficult, and many existing results are mostly on the synchronous consensus algorithm [13-15], in which self-delays equaling to the corresponding communication delays are introduced for each agent in the coordination control part. Compared with the first-order multi-agent systems, the consensus algorithm without any self-delay, which is called asynchronous consensus algorithm, has not been studied extensively for the second-order multi-agent systems. Using small- $\mu$ stability theorem, Yang et al. [16] obtained the frequency-domain 
consensus conditions for the second-order multi-agent systems with time-varying communication delays. Based on frequency-domain analysis [17] and Lyapunov-Krasovskii functional method [18], Spong et al. proved that, by choosing proper consensus protocol and control parameters, the second-order multiagent systems with heterogeneous communication delays can converge to a stationary consensus without any relationship to the delays. Using the properties of nonnegative matrices, Lin and Jia [19] obtained delay-independent sufficient conditions for the second-order discrete-time multi-agent systems with heterogeneous communication delays converging to the stationary consensus under dynamically changing topologies.

To our knowledge, however, the consensus problem under input delays has not attracted much more attention. In some reports, the identical communication delay introduced in the synchronous consensus algorithm can be treated as the identical input delay $[2,14,15]$. Using frequency-domain analysis method, Tian and Liu [5] considered the consensus problem of the first-order multi-agent systems with heterogeneous input delays based on undirected graphs, and obtained the decentralized consensus criterion depending on the input delays. Moreover, the decentralized consensus condition, which depends only on the input delays, is also obtained for the first-order multi-agent systems with both heterogeneous communication delays and input delays based on the digraph in [5]. In [20], Tian and Liu investigated the leader-following consensus problem of the second-order multi-agent systems with heterogeneous input delays and symmetric coupling weights, and the decentralized consensus condition with some prerequisites is obtained for the system converging to the states of the dynamic leader asymptotically. Furthermore, the robustness of the symmetric system with asymmetric weight perturbation is also investigated in [20], and a bound of the largest singular value of the perturbation matrix is obtained as the robust consensus condition.

In this paper, we consider the consensus problem of second-order multi-agent systems with velocity damping term in the agent's dynamics, and analyze the consensus conditions for the system with heterogeneous delays converging to the stationary consensus. Firstly, we investigate the consensus problem for the system based on undirected and symmetric graph with heterogeneous input delays, and a decentralized consensus condition, which is delay-dependent, is obtained by using some early results for the Internet congestion control with heterogeneous communication delays [21]. Then, we study the consensus problem for the system based on general directed graph with both heterogeneous input delays and communication delays by using Greshgorin disc theorem, and another decentralized consensus condition, which depends on the input delays only, is also obtained. This consensus condition is more conservative than the former for the existence of heterogeneous communication delays and the asymmetry of coupling weights, but it can be applied to the systems based on directed graph with asymmetric weights.

\section{Preliminaries on Graph Theory}

A weighted directed graph (digraph) $\mathcal{G}=(\mathcal{V}, \mathcal{E}, \mathcal{A})$ of order $n$ consists of a set of vertices $\mathcal{V}=\{1, \ldots, n\}$, a set of edges $\mathcal{E} \subseteq \mathcal{V} \times \mathcal{V}$ and a weighted adjacency matrix $\mathcal{A}=\left[a_{i j}\right] \in R^{n \times n}$ with nonnegative adjacency elements $a_{i j}$. The node indexes belong to a finite index set $\mathcal{J}=\{1,2, \ldots, n\}$. An edge of the weighted diagraph $\mathcal{G}$ is denoted by $e_{i j}=(i, j) \in \mathcal{E}$, i.e., $e_{i j}$ is a directed edge from $i$ to $j$. We assume that the adjacency elements associated with the edges of the digraph are positive, i.e., $a_{i j}>0 \Leftrightarrow e_{i j} \in \mathcal{E}$. Moreover, we assume $a_{i i}=0$ for all $i \in \mathcal{J}$. The set of neighbors of node $i$ is denoted by $N_{i}=\{j \in \mathcal{V}:(i, j) \in \mathcal{E}\}$. In the digraph $\mathcal{G}$, if $(i, j) \in \mathcal{E} \leftrightarrow(j, i) \in \mathcal{E}$, we usually say $\mathcal{G}$ is undirected graph or bidirectional graph. The out-degree of node $i$ is defined as: $\operatorname{deg}_{\text {out }}(i)=\sum_{j=1}^{n} a_{i j}$. Let $\mathcal{D}$ be the diagonal matrix with the out-degree of each node along the diagonal and call it the degree matrix of $\mathcal{G}$. The Laplacian matrix of the weighted digraph is defined as $L=\mathcal{D}-\mathcal{A}$.

If there is a path in $\mathcal{G}$ from one node $i$ to another node $j$, then $j$ is said to be reachable from $i$. If not, then $j$ is said to be not reachable from $i$. If a node is reachable from every other node in the digraph, then 
we say it globally reachable. A digraph is strongly connected if every node in the digraph is globally reachable. An undirected graph is connected if it contains a globally reachable node.

\section{Problem Formulation}

In a multi-agent system composed of $n$ agents, each agent can be regarded as a node in a digraph, and information flow between neighboring agents can be considered as directed paths between the nodes in the digraph. Thus, the interconnection topology of multi-agent systems can be described as a diagraph $\mathcal{G}=(\mathcal{V}, \mathcal{E}, \mathcal{A})$. In this paper, we just consider static topology $\mathcal{G}$, i.e., the connection of the nodes in the diagraph $\mathcal{G}$ does not change with time.

Consider the second-order dynamic agents modeled by

$$
\begin{aligned}
\dot{x}_{i} & =v_{i}, \\
m_{i} \dot{v}_{i} & =F_{i}, i \in \mathcal{J},
\end{aligned}
$$

where $x_{i} \in R$ and $v_{i} \in R$ are the position and the velocity of the agent $i$ respectively, $m_{i}>0$ is the mass of the agent $i$, and $F_{i}$ is the total force on the agent $i$. Analogous to [22], the total force $F_{i}$ in the second-order model (1) consists of two components:

$$
F_{i}=-\alpha_{i} v_{i}+u_{i}
$$

where $u_{i}$ is the external control input, and $-\alpha_{i} v_{i}$ with $\alpha_{i}>$ o denotes the velocity damping term caused by the resistance, e.g., the friction. Then, the second-order model (1) becomes

$$
\begin{aligned}
\dot{x}_{i} & =v_{i}, \\
m_{i} \dot{v}_{i} & =-\alpha_{i} v_{i}+u_{i}, i \in \mathcal{J} .
\end{aligned}
$$

With non-negligible input delays for the external control, the agents (2) become

$$
\begin{aligned}
\dot{x}_{i}(t) & =v_{i}(t), \\
m_{i} \dot{v}_{i}(t) & =-\alpha_{i} v_{i}(t)+u_{i}\left(t-T_{i}\right), i \in \mathcal{J},
\end{aligned}
$$

where $T_{i}>0$ is the input delay of the agent $i$. For the system (3), we take a consensus protocol based on the agents' position states as follows

$$
u_{i}=\kappa_{i} \sum_{j \in N_{i}} a_{i j}\left(x_{j}-x_{i}\right),
$$

where $\kappa_{i}>0, N_{i}$ denotes the neighbors of agent $i$, and $a_{i j}>0$ is the adjacency element of $\mathcal{A}$ in the digraph $\mathcal{G}=(\mathcal{V}, \mathcal{E}, \mathcal{A})$. Under the communication delays, the protocol (4) becomes

$$
u_{i}(t)=\kappa_{i} \sum_{j \in N_{i}} a_{i j}\left(x_{j}\left(t-\tau_{i j}\right)-x_{i}(t)\right),
$$

where $\tau_{i j}$ is the communication delay from agent $j$ to agent $i$.

With the protocol (5), the closed-loop form of the system (3) is

$$
\begin{aligned}
\dot{x}_{i}(t) & =v_{i}(t), \\
m_{i} \dot{v}_{i}(t) & =-\alpha_{i} v_{i}(t)+\kappa_{i} \sum_{j \in N_{i}} a_{i j}\left(x_{j}\left(t-T_{i}-\tau_{i j}\right)-x_{i}\left(t-T_{i}\right)\right), i \in \mathcal{J} .
\end{aligned}
$$

Remark 1. In [20], Tian and Liu has studied the leader-following consensus problem of the second-order multi-agent systems with heterogeneous input delays under double-consensus algorithm, and obtained the consensus conditions for the system with symmetric and asymmetric weights respectively. Different from [20], we consider the stationary consensus of the second-order dynamic agents (6) with velocity damping term, and analyze the consensus conditions for the system with heterogeneous communication delays and input delays. 


\section{Consensus Criterion}

\subsection{Consensus under Heterogeneous Input Delays}

In this section, we investigate the consensus problem of multi-agent systems (6) just with heterogeneous input delays only as follows

$$
\begin{aligned}
\dot{x}_{i}(t) & =v_{i}(t), \\
m_{i} \dot{v}_{i}(t) & =-\alpha_{i} v_{i}(t)+\kappa_{i} \sum_{j \in N_{i}} a_{i j}\left(x_{j}\left(t-T_{i}\right)-x_{i}\left(t-T_{i}\right)\right), i \in \mathcal{J} .
\end{aligned}
$$

Firstly, we give an assumption on the velocity damping coefficient $\alpha_{i}$, the mass $m_{i}$ and input delay $T_{i}$ in the following.

Assumption 2. $\left(T_{i} \frac{\alpha_{i}}{m_{i}}-T_{j} \frac{\alpha_{j}}{m_{j}}\right)\left(T_{i}-T_{j}\right) \leq 0, \forall i, j \in \mathcal{J}, i \neq j$.

Now, we present some sufficient conditions for second-order multi-agent systems with heterogeneous input delays.

Theorem 3. Consider the network of $n$ dynamic agents (7) with a static interconnection topology $9=$ $(\mathcal{V}, \mathcal{E}, \mathcal{A})$ that is undirected (or bidirectional) and connected, and the topology graph has symmetric weights, i.e., $a_{i j}=a_{j i}$. Then, under Assumption 2, all the agents in system (7) asymptotically converge to a stationary consensus, i.e., $\lim _{t \rightarrow \infty} x_{i}(t)=c, \lim _{t \rightarrow} v_{i}(t)=0, \forall i \in \mathcal{J}$, where $c$ is a constant, if

$$
\sum_{j \in N_{i}} a_{i j}<\frac{m_{i}}{2 \kappa_{i}\left(G_{i}^{M}\right)^{-1}}, \forall i \in \mathcal{J}
$$

where $G_{i}^{M}$ is the gain margin of the transfer function $W_{i}(s)=\frac{\mathrm{e}^{-s T_{i}}}{s^{2}+\frac{\alpha_{i}}{m_{i}} s}$.

Before proving Theorem 3, we list two useful lemmas as follows.

Lemma 4. [23] Let $Q \in C^{n \times n}, Q=Q^{\star} \geq 0$ and $T=\operatorname{diag}\left\{t_{i}, t_{i} \in C\right\}$. Then

$$
\lambda(Q T) \in \rho(Q) \operatorname{Co}\left(\mathrm{o} \cup\left\{t_{i}\right\}\right)
$$

where $\lambda(\cdot)$ denotes matrix eigenvalue, $\rho(\cdot)$ denotes the matrix spectral radius, and $\mathrm{Co}(\cdot)$ denotes the convex hull.

Based on Remark 4 and Claim 1 in [21], we obtain the following lemma.

Lemma 5. Suppose that Assumption 2 holds for the frequency response of a family of systems described by

$$
G_{i}(\mathrm{j} \omega)=\frac{G_{i}^{M}}{s^{2}+\mathrm{j} \frac{\alpha_{i}}{m_{i}} \omega} \mathrm{e}^{-\mathrm{j} T_{i} \omega}, \quad i \in \mathcal{J},
$$

where $G_{i}^{M}$ is the gain margin of the transfer function $W_{i}(s)=\frac{\mathrm{e}^{-s T_{i}}}{s^{2}+\frac{\alpha_{i}}{m_{i}} s}$. Then, $\gamma \operatorname{Co}\left(\mathrm{o} \cup\left\{G_{i}(\mathrm{j} \omega), i \in \mathcal{J}\right\}\right)$ does not contain the point $(-1, \mathrm{jo})$ for any given real number $\gamma \in[0,1)$ and any $\omega \in(-\infty, \infty)$.

Now, we give the proof of Theorem 3.

The system (7) is rewritten as follows

$$
\begin{aligned}
\dot{x}_{i}(t) & =v_{i}(t), \\
\dot{v}_{i}(t) & =-\bar{\alpha}_{i} v_{i}(t)+\bar{\kappa}_{i} \sum_{j \in N_{i}} a_{i j}\left(x_{j}\left(t-T_{i}\right)-x_{i}\left(t-T_{i}\right)\right), i \in \mathcal{J},
\end{aligned}
$$


where $\bar{\alpha}_{i}=\frac{\alpha_{i}}{m_{i}}$ and $\bar{\kappa}_{i}=\frac{\kappa_{i}}{m_{i}}$. Taking the Laplace transform of the system (9), we obtain the characteristic equation of the system (9) about $x(t)=\left[x_{1}(t), \cdots, x_{n}(t)\right]^{T}$ as follows

$$
\operatorname{det}\left(\operatorname{diag}\left\{s^{2}+\bar{\alpha}_{i} s, i \in \mathcal{J}\right\}+\operatorname{diag}\left\{\bar{\kappa}_{i} \mathrm{e}^{-T_{i} s}, i \in \mathcal{J}\right\} L\right)=0 .
$$

Define $D(s)=\operatorname{det}\left(\operatorname{diag}\left\{s^{2}+\bar{\alpha}_{i} s, i \in \mathcal{J}\right\}+\operatorname{diag}\left\{\bar{\kappa}_{i} \mathrm{e}^{-T_{i} s}, i \in \mathcal{J}\right\} L\right)$, and we will prove that all the zeros of $D(s)$ are on the open left half complex plane or $s=0$ in the following.

When $s=0, D(\mathrm{o})=\operatorname{det}\left(\operatorname{diag}\left\{0^{2}+\bar{\alpha}_{i} \mathrm{O}, i \in \mathcal{J}\right\}+\operatorname{diag}\left\{\bar{\kappa}_{i} \mathrm{e}^{-T_{i} \mathrm{O}}, i \in \mathcal{J}\right\} L\right)=\operatorname{det}\left(\operatorname{diag}\left\{\bar{\kappa}_{i}, i \in \mathcal{J}\right\}\right) \operatorname{det}(L)$, Because the interconnection graph $\mathcal{G}=(\mathcal{V}, \mathcal{E}, \mathcal{A})$ is connected, o is a simple eigenvalue of $L$ [24], i.e., $\operatorname{rank}(L)=n-1$. Hence, $D(s)$ has only one zero at $s=0$.

When $s \neq 0$, define $F(s)=\operatorname{det}\left(I+\operatorname{diag}\left\{\frac{\bar{\kappa}_{i}}{s^{2}+\bar{\alpha}_{i} s} \mathrm{e}^{-T_{i} s}, i \in \mathcal{J}\right\} L\right)$. We will prove that the zeros of $F(s)$ lie on the open left half complex plane. According to the General Nyquist stability criterion [25], the zeros of $F(s)$ are on the open left half complex plane, if $\lambda\left(\operatorname{diag}\left\{\frac{\bar{\kappa}_{i}}{(\mathrm{j} \omega)^{2}+\mathrm{j} \omega \bar{\alpha}_{i}} \mathrm{e}^{-\mathrm{j} \omega T_{i}}, i \in \mathcal{J}\right\} L\right)$ does not enclose the point $(-1$, jo $)$ for $\omega \in R$.

For the symmetric weights $\left(a_{i j}=a_{j i}\right)$, we get $L=L^{T} \geq 0$ according to the definition of the Laplacian matrix. Based on Lemma 4, we get

$$
\begin{aligned}
& \lambda\left(\operatorname{diag}\left\{\frac{\bar{\kappa}_{i}}{-\omega^{2}+\mathrm{j} \bar{\alpha}_{i} \omega} \mathrm{e}^{-\mathrm{j} T_{i} \omega}\right\} L\right) \\
= & \lambda\left(\operatorname{diag}\left\{\frac{G_{i}^{M}}{-\omega^{2}+\mathrm{j} \bar{\alpha}_{i} \omega} \mathrm{e}^{-\mathrm{j} T_{i} \omega} \operatorname{diag}\left\{\sqrt{\bar{\kappa}_{i}\left(G_{i}^{M}\right)^{-1}}\right\} L \operatorname{diag}\left\{\sqrt{\bar{\kappa}_{i}\left(G_{i}^{M}\right)^{-1}}\right\}\right)\right. \\
\in & \rho\left(\operatorname{diag}\left\{\sqrt{\bar{\kappa}_{i}\left(G_{i}^{M}\right)^{-1}}\right\} L \operatorname{diag}\left\{\sqrt{\bar{\kappa}_{i}\left(G_{i}^{M}\right)^{-1}}\right\}\right) \operatorname{Co}\left(\mathrm{o} \cup \frac{G_{i}^{M}}{-\omega^{2}+\mathrm{j} \bar{\alpha}_{i} \omega} \mathrm{e}^{-\mathrm{j} T_{i} \omega}\right) .
\end{aligned}
$$

Since the spectral radius of any matrix is bounded by its largest absolute row sum, it follows from the condition (8) that

$$
\begin{aligned}
\rho\left(\operatorname{diag}\left\{\sqrt{\bar{\kappa}_{i}\left(G_{i}^{M}\right)^{-1}}\right\} \operatorname{diag}\left\{\sqrt{\left.\bar{\kappa}_{i}\left(G_{i}^{M}\right)^{-1}\right\}}\right)\right. & =\rho\left(\operatorname{diag}\left\{\bar{\kappa}_{i}\left(G_{i}^{M}\right)^{-1}\right\} L\right) \\
& \leq \max _{i \in \mathcal{J}} \bar{\kappa}_{i}\left(G_{i}^{M}\right)^{-1}\left(2 \sum_{j \in N_{i}} a_{i j}\right) \\
& <1 .
\end{aligned}
$$

Therefore, from Lemma 5, we obtain that

$$
(-1,0) \notin \rho\left(\operatorname{diag}\left\{\sqrt{\bar{\kappa}_{i}\left(G_{i}^{M}\right)^{-1}}\right\} \operatorname{Ldiag}\left\{\sqrt{\bar{\kappa}_{i}\left(G_{i}^{M}\right)^{-1}}\right\}\right) \operatorname{Co}\left(\mathrm{o} \cup \frac{G_{i}^{M}}{-\omega^{2}+\mathrm{j} \bar{\alpha}_{i} \omega} \mathrm{e}^{-\mathrm{j} T_{i} \omega}\right),
$$

i.e., $\lambda\left(\operatorname{diag}\left\{\frac{\bar{\kappa}_{i}}{\mathrm{j} \omega)^{2}+\mathrm{j} \omega \bar{\alpha}_{i}} \mathrm{e}^{-\mathrm{j} \omega T_{i}}, i \in \mathcal{J}\right\} L\right)$ does not enclose the point $(-1, \mathrm{jo})$ for $\omega \in R$, which implies that the zeros of $F(s)$ are all on the open left half complex plane.

Now, we have proved that $D(s)$ has its zeros on the open left half complex plane except for one zero at $s=0$. Thus, the state $x_{i}(t)$ of the system (7) converges to a steady state, i.e., $\lim _{t \rightarrow \infty} x_{i}(t)=x_{i}^{*}, i \in \mathcal{J}$, and $\lim _{t \rightarrow \infty} v_{i}(t)=0, \forall i \in \mathcal{J}$ holds for (7). It is obtained from (7) that $L\left[x_{1}^{*}, \cdots, x_{n}^{*}\right]^{T}=0$. Since $\operatorname{rank}(L)=n-1$ and $L[1, \cdots, 1]^{T}=0$ from the definition of the Laplacian matrix $L$, the roots of $L x^{*}=0$ can be expressed as $x^{*}=c[1, \cdots, 1]^{T}$, where $c$ is a constant. Theorem 3 is proved.

Remark 6. Obviously, the consensus condition (8) in Theorem 3 depends strictly on the Assumption 2 and the symmetry of the coupling weights between agents. 


\subsection{Consensus under Heterogeneous Input and Communication Delays}

In multi-agent systems, the interconnection topology composed of dynamic agents is usually asymmetric, and the communication delays caused by information transmission always exist between neighboring agents. Thus, the Lemma 4 and Lemma 5 which play important roles in the proof of Theorem 3 cannot be applied in these cases. In this section, we will analyze the consensus of the second-order dynamic agents (6) with both heterogeneous input delays and communication delays under general directed interconnection topology.

Theorem 7. Consider the network of $n$ dynamic agents (6) with a static interconnection topology $\mathcal{G}=$ $(\mathcal{V}, \mathcal{E}, \mathcal{A})$ that has a globally reachable node. If

$$
\sum_{j \in N_{i}} a_{i j}<\frac{\alpha_{i}^{2}}{2 \kappa_{i}\left(m_{i}+\alpha_{i} T_{i}\right)}, \forall i \in \mathcal{J},
$$

all the agents in the system (6) converge to a stationary consensus asymptotically.

Proof: Firstly, rewrite the system (6) as

$$
\begin{aligned}
& \dot{x}_{i}(t)=v_{i}(t), \\
& \dot{v}_{i}(t)=-\bar{\alpha}_{i} v_{i}(t)+\bar{\kappa}_{i} \sum_{j \in N_{i}} a_{i j}\left(x_{j}\left(t-T_{i}-\tau_{i j}\right)-x_{i}\left(t-T_{i}\right)\right), i \in \mathcal{J},
\end{aligned}
$$

where $\bar{\alpha}_{i}=\frac{\alpha_{i}}{m_{i}}$ and $\bar{\kappa}_{i}=\frac{\kappa_{i}}{m_{i}}$. Taking the Laplace transform of the system (12), we obtain that the characteristic equation of the system (12) about $x(t)=\left[x_{1}(t), \cdots, x_{n}(t)\right]^{T}$ is

$$
\operatorname{det}\left(\operatorname{diag}\left\{s^{2}+\bar{\alpha}_{i} s, i \in \mathcal{J}\right\}+\operatorname{diag}\left\{\bar{\kappa}_{i} \mathrm{e}^{-T_{i} s}, i \in \mathcal{J}\right\} L(s)\right)=0,
$$

where the $n \times n$ matrix $L(s)=\left\{l_{i j}(s)\right\}$ is defined by

$$
l_{i j}(s)= \begin{cases}-a_{i j} \mathrm{e}^{-\tau_{i j} s}, & j \in N_{i} \\ \sum_{j \in N_{i}} a_{i j}, & j=i \\ 0, & \text { otherwise }\end{cases}
$$

and $L(\mathrm{o})=L$, which is the Laplacian matrix.

Define $\tilde{D}(s)=\operatorname{det}\left(\operatorname{diag}\left\{s^{2}+\bar{\alpha}_{i} s, i \in \mathcal{J}\right\}+\operatorname{diag}\left\{\bar{\kappa}_{i} \mathrm{e}^{-T_{i} s}, i \in \mathcal{J}\right\} L(s)\right)$, and we will prove that all the zeros of $\tilde{D}(s)$ are on the open left half plane or $s=0$ in the following.

When $s=0, \tilde{D}(\mathrm{o})=\operatorname{det}\left(\operatorname{diag}\left\{0^{2}+\bar{\alpha}_{i} \mathrm{O}, i \in \mathcal{J}\right\}+\operatorname{diag}\left\{\bar{\kappa}_{i} \mathrm{e}^{-T_{i 0} 0}, i \in \mathcal{J}\right\} L(\mathrm{o})\right)=\operatorname{det}\left(\operatorname{diag}\left\{\bar{\kappa}_{i}, i \in \mathcal{J}\right) \operatorname{det}(L)\right.$, Because the interconnection topology $\mathcal{G}=(\mathcal{V}, \mathcal{E}, \mathcal{A})$ has a globally reachable node, o is a simple eigenvalue of $L$ [24]. Hence, $\tilde{D}($ o $)=$ o, i.e., $\tilde{D}(s)$ has only one zero at $s=0$.

When $s \neq 0$, define $\tilde{F}(s)=\operatorname{det}\left(I+\operatorname{diag}\left\{\frac{\bar{\kappa}_{i}}{s^{2}+\bar{\alpha}_{i} s} \mathrm{e}^{-T_{i} s}, i \in \mathcal{J}\right\} L(s)\right)$. We will prove the zeros of $\tilde{F}(s)$ lie on the open left half complex plane. According to the General Nyquist stability criterion [25], the zeros of $\tilde{F}(s)$ are on the open left half complex plane, if $\lambda\left(\operatorname{diag}\left\{\frac{\bar{\kappa}_{i}}{(\mathrm{j} \omega)^{2}+\mathrm{j} \omega \bar{\alpha}_{i}} \mathrm{e}^{-\mathrm{j} \omega T_{i}}, i \in \mathcal{J}\right\} L(\mathrm{j} \omega)\right)$ does not enclose the point $(-1$, jo $)$ for $\omega \in R$. Based on the Greshgorin's disc theorem,

$$
\begin{aligned}
& \lambda\left(\operatorname{diag}\left\{\frac{\bar{\kappa}_{i}}{-\omega^{2}+\mathrm{j} \bar{\alpha}_{i} \omega} \mathrm{e}^{-\mathrm{j} T_{i} \omega}, i \in \mathcal{J}\right\} L(s)\right) \\
\in & \bigcup_{i \in \mathcal{J}}\left\{\zeta: \zeta \in C,\left|\zeta-\frac{\bar{\kappa}_{i}\left(\sum_{j \in N_{i}} a_{i j}\right)}{-\omega^{2}+\mathrm{j} \bar{\alpha}_{i} \omega} \mathrm{e}^{-\mathrm{j} T_{i} \omega}\right| \leq \sum_{j \in N_{i}}\left|\frac{\bar{\kappa}_{i} a_{i j}}{-\omega^{2}+\mathrm{j} \bar{\alpha}_{i} \omega} \mathrm{e}^{-\mathrm{j}\left(T_{i}+\tau_{i j}\right) \omega}\right|\right\} \\
= & \bigcup_{i \in \mathcal{J}}\left\{\zeta: \zeta \in C,\left|\zeta-\frac{\bar{\kappa}_{i} g_{i}}{-\omega^{2}+\mathrm{j} \bar{\alpha}_{i} \omega} \mathrm{e}^{-\mathrm{j} T_{i} \omega}\right| \leq\left|\frac{\bar{\kappa}_{i} g_{i}}{-\omega^{2}+\mathrm{j} \bar{\alpha}_{i} \omega} \mathrm{e}^{-\mathrm{j} T_{i} \omega}\right|\right\}
\end{aligned}
$$


holds for $\omega \in R$, where $g_{i}=\sum_{j \in N_{i}} a_{i j}$.

Then, $\lambda\left(\operatorname{diag}\left\{\frac{\bar{\kappa}_{i}}{(\mathrm{j} \omega)^{2}+\mathrm{j} \omega \bar{\alpha}_{i}} \mathrm{e}^{-\mathrm{j} \omega T_{i}}, i \in \mathcal{J}\right\} L(\mathrm{j} \omega)\right)$ does not enclose the point $(-1, \mathrm{jo})$ for $\omega \in R$ as long as the point $(-a, \mathrm{jo})$ with $a \geq 1$ does not in the $\operatorname{disc}\left\{\zeta: \zeta \in C,\left|\zeta-\frac{\bar{\kappa}_{i} g_{i}}{-\omega^{2}+\mathrm{j} \bar{\alpha}_{i} \omega} \mathrm{e}^{-\mathrm{j} T_{i} \omega}\right| \leq\left|\frac{\bar{\kappa}_{i} g_{i}}{-\omega^{2}+\mathrm{j} \bar{\alpha}_{i} \omega} \mathrm{e}^{-\mathrm{j} T_{i} \omega}\right|\right\}$ for all $\omega \in R$, i.e., $\left|-a+\mathrm{jo}-\frac{\bar{\kappa}_{i} g_{i}}{-\omega^{2}+\mathrm{j} \bar{\alpha}_{i} \omega} \mathrm{e}^{-\mathrm{j} T_{i} \omega}\right|>\left|\frac{\bar{\kappa}_{i} g_{i}}{-\omega^{2}+\mathrm{j} \bar{\alpha}_{i} \omega} \mathrm{e}^{-\mathrm{j} T_{i} \omega}\right|$ holds for all $\omega \in R$ when $a \geq 1$.

By calculating, we obtain

$$
\left|-a+\mathrm{jo}-\frac{\bar{\kappa}_{i} g_{i}}{-\omega^{2}+\mathrm{j} \bar{\alpha}_{i} \omega} \mathrm{e}^{-\mathrm{j} T_{i} \omega}\right|^{2}-\left|\frac{\bar{\kappa}_{i} g_{i}}{-\omega^{2}+\mathrm{j} \bar{\alpha}_{i} \omega} \mathrm{e}^{-\mathrm{j} T_{i} \omega}\right|^{2}=a\left(a-2 \bar{\kappa}_{i} g_{i} \frac{\cos \left(\omega T_{i}\right)+\bar{\alpha}_{i} \frac{\sin \left(\omega T_{i}\right)}{\omega}}{\omega^{2}+\bar{\alpha}_{i}^{2}}\right) .
$$

Because $\cos \left(\omega T_{i}\right) \leq 1$ and $\frac{\sin \left(\omega T_{i}\right)}{\omega} \leq T_{i}$ hold for $\omega \in R$, it follows from (11) that

$$
2 \bar{\kappa}_{i} g_{i} \frac{\cos \left(\omega T_{i}\right)+\bar{\alpha}_{i} \frac{\sin \left(\omega T_{i}\right)}{\omega}}{\omega^{2}+\bar{\alpha}_{i}^{2}} \leq \frac{2 \bar{\kappa}_{i} g_{i}\left(1+\bar{\alpha}_{i} T_{i}\right)}{\bar{\alpha}_{i}^{2}}<1 .
$$

Hence, $\left|-a+\mathrm{jo}-\frac{\bar{\kappa}_{i} g_{i}}{-\omega^{2}+\mathrm{j} \bar{\alpha}_{i} \omega} \mathrm{e}^{-\mathrm{j} T_{i} \omega}\right|>\left|\frac{\bar{\kappa}_{i} g_{i}}{-\omega^{2}+\mathrm{j} \bar{\alpha}_{i} \omega} \mathrm{e}^{-\mathrm{j} T_{i} \omega}\right|$ holds for all $\omega \in R$ when $a \geq 1$.

Now, we have proved that $\tilde{D}(s)$ has its zeros on the open left half complex plane except for a zero at $s=0$. Thus, the state $x_{i}(t)$ of the system (6) converges to a steady state, i.e., $\lim _{t \rightarrow \infty} x_{i}(t)=x_{i}^{*}, i \in \mathcal{J}$, and $\lim _{t \rightarrow \infty} v_{i}(t)=0, \forall i \in \mathcal{J}$ holds for (6). Then, analogous to the proof of Theorem 3, the system (6) converges to a stationary consensus for the digraph that has a globally reachable node. Theorem 7 is proved.

Remark 8. In the networks composed of interconnected dynamic systems, the scalability is a important property that needs to be maintained $[5,20,21,23]$. Obviously, the decentralized consensus conditions (8) and (11) maintain the scalability of the multi-agent network. Without having to redesign the entire network whenever an agent is added or removed, the networks (6) and (7) can achieve the desired collective behavior as long as the local conditions for the agent and its neighbors hold respectively, and the connectedness of the interconnection topology is maintained.

Remark 9. In the consensus analysis of the multi-agent systems, Greshgorin's disc theorem has been extensively used to obtain the communication delay-independent consensus condition for the system with heterogeneous communication delays [8,17]. In [17], decentralized frequency-domain consensus conditions have been obtained for the multi-agent systems with agents' dynamic modeled by strictly stable linear systems under heterogeneous communication delays. Then, by transformation, the system (6) can be expressed as a special case of the system studied in [17]. However, (11) gives a concrete algebraic criterion, which is convenient for the design of the consensus algorithm.

Remark 10. According to [20] (the inequality (24) there in), the consensus condition (8) in Theorem 3 satisfies

$$
\frac{m_{i}}{2 \kappa_{i}\left(G_{i}^{M}\right)^{-1}}>\frac{\alpha_{i}}{2 \kappa_{i} T_{i}}>\frac{\alpha_{i}^{2}}{2 \kappa_{i}\left(m_{i}+\alpha_{i} T_{i}\right)} .
$$

Thus, under the same conditions, the consensus condition (11) in Theorem 7 is more conservative than the consensus condition (8) given in Theorem 3.

\section{Simulation}

Example 11. Consensus under input delays based on symmetric graph.

Consider a multi-agent network of five dynamic agents described by (7). The interconnection topology is described in Figure 1, and the graph is undirected and connected. The symmetric weights of the edges are: $a_{12}=a_{21}=0.2, a_{15}=a_{51}=0.3, a_{23}=a_{3^{2}}=0.5, a_{24}=a_{4^{2}}=0.1, a_{45}=a_{54}=0.4$. The input delays of the agents are: $T_{1}=0.5(s), T_{2}=0.6(s), T_{3}=0.8(s), T_{4}=0.4(s)$ and $T_{5}=0.3(s)$. The velocity 
damping coefficients of the agents are: $\alpha_{1}=1, \alpha_{2}=\frac{2}{3}, \alpha_{3}=0.25, \alpha_{4}=2, \alpha_{5}=3$, and the mass of each agent is assumed to be 1, i.e., $m_{i}=1, i=1, \cdots, 5$. Thus, the Assumption 2 holds for all the agents. For the transfer functions $W_{i}(s)=\frac{\mathrm{e}^{-s T_{i}}}{s^{2}+\frac{\alpha_{i}}{m_{i}} s}, i=1, \cdots, 5$, by using the Matlab simulator, we obtain the gain margins: $G_{1}^{M} \simeq 2.15, G_{2}^{M} \simeq 1.18, G_{3}^{M} \simeq 0.32, G_{4}^{M} \simeq 5.56$ and $G_{5}^{M} \simeq 11.24$. According to the condition (8), we obtain that the control parameters $\kappa_{i}$ satisfy: $\kappa_{1} \in(0,2.15), \kappa_{2} \in(0,0.74), \kappa_{3} \in(0,0.32), \kappa_{4} \in(0,5.56)$, $\kappa_{5} \in(0,8.03)$, and we choose $\kappa_{1}=2, \kappa_{2}=0.7, \kappa_{3}=0.3, \kappa_{4}=2$ and $\kappa_{5}=3$. Then, with the initial states generated randomly, the agents in the system (7) converge to a stationary consensus (see Figure 2).

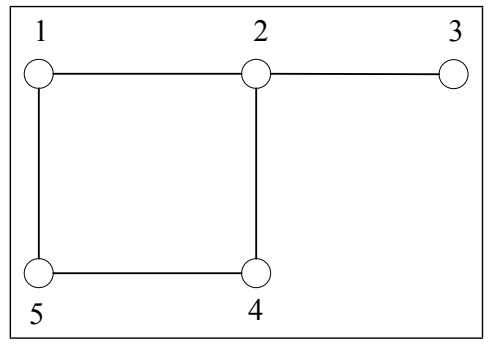

Figure 1: Undirected graph with symmetric weights

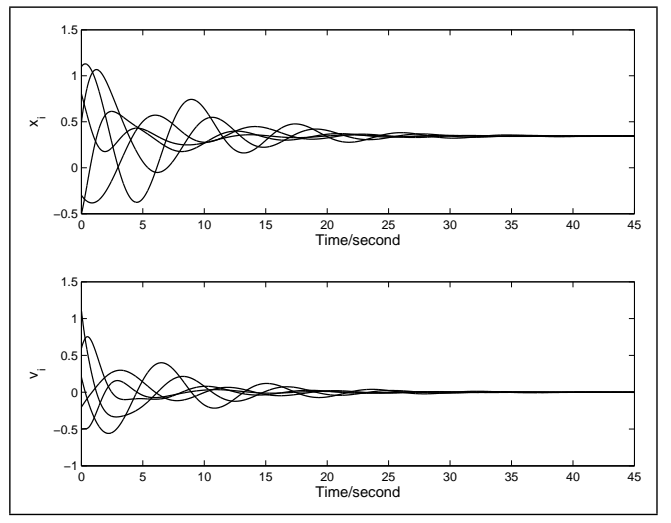

Figure 2: Consensus convergence under input delays

Example 12. Consensus under input and communication delays based on asymmetric digraph.

Consider a network of five agents described by (6). The interconnection topology is a digraph described in Figure 3, and the globally reachable node set of the digraph is $\{3,4\}$. The weights of the directed edges are: $a_{12}=0.2, a_{24}=0.2, a_{34}=0.5, a_{43}=0.1, a_{54}=0.4, a_{51}=0.1$, and the corresponding communication delays are: $\tau_{12}=0.15(\mathrm{~s}), \tau_{24}=0.2(\mathrm{~s}), \tau_{34}=0.5(\mathrm{~s}), \tau_{43}=0.3(\mathrm{~s}), \tau_{54}=0.4(\mathrm{~s})$, $\tau_{5_{1}}=0.2(s)$. The velocity damping coefficients of the agents are: $\alpha_{1}=1.5, \alpha_{2}=2, \alpha_{3}=1, \alpha_{4}=2$, $\alpha_{5}=3$, and the mass of each agent is assumed to be 1 . Choosing the control parameters: $\kappa_{1}=1, \kappa_{2}=2$, $\kappa_{3}=0.5, \kappa_{4}=2, \kappa_{5}=3$, we obtain from the condition (11) that the constraints on the input delays are: $T_{1} \in(0,1.83)(s), T_{2} \in(0,2)(s), T_{3} \in(0,1)(s), T_{4} \in(0,4.5)(s)$ and $T_{5} \in(0,0.67)(s)$. With $T_{1}=1(s)$, $T_{2}=0.8(s), T_{3}=0.6(s), T_{4}=2(s), T_{5}=0.6(s)$, the agents in the system (6) converge to a stationary consensus (see Figure 4).

\section{Conclusions}

In this paper, we investigate the consensus problem of second-order multi-agent systems with velocity damping term in the agent's dynamic. Based on the frequency-domain analysis, two sufficient 


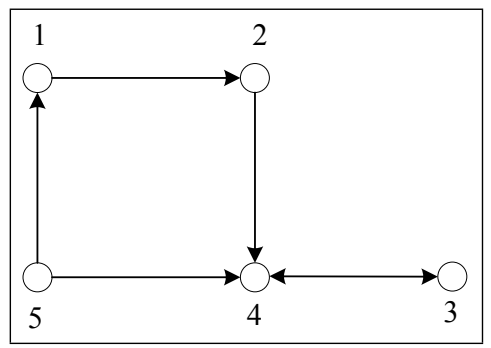

Figure 3: Digraph composed of 5 agents

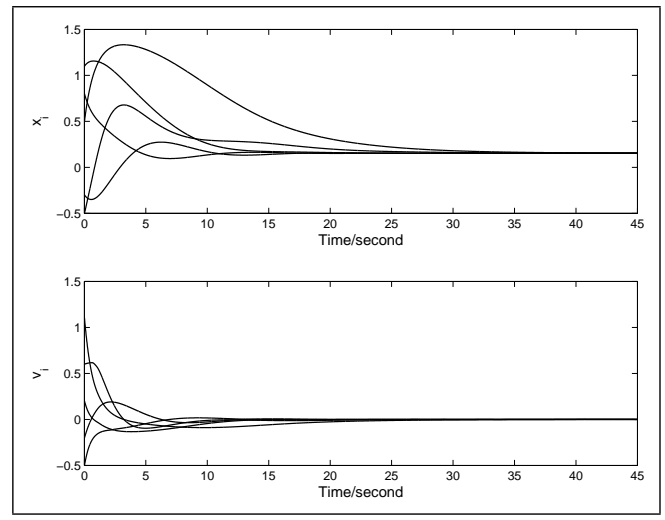

Figure 4: Consensus convergence under input and communication delays

decentralized consensus conditions are obtained. One consensus condition is for the system with heterogeneous input delays based on undirected and symmetric graph, and is dependent on the input delays. The other consensus condition is for the system with both heterogeneous input delays and communication delays based on general directed graph, and depends on the input delays only. Although the later consensus condition is more conservative than the former, it can be applied to the systems based on directed graph with asymmetric coupling weights.

\section{Acknowledgements}

This work was supported by Specialized Research Fund for the Doctoral Program of Higher Education of China (Grant No. 20090093120006).

\section{Bibliography}

[1] A. Jadbabaie, J. Lin, A.S. Morse, Coordination of Groups of Mobile Autonomous Agents Using Nearest Neighbor Rules, IEEE Transactions on Automatic Control, 48(6):988-1001, 2003.

[2] R. Olfati-Saber, R. Murray, Consensus Problems in Networks of Agents with Switching Topology and Time-delays, IEEE Transactions on Automatic Control, 49(9):1520-1533, 2004.

[3] W. Ren, E. Atkins, Distributed Multi-vehicle Coordinated Control via Local Information Exchange, International Journal of Robust and Nonlinear Control, 17(10-11):1002-1033, 2007.

[4] Y. Hong, L. Gao, D. Cheng, J. Jiang, Lyapunov-based Approach to Multiagent Systems with Switching Jointly Connected Interconnection, IEEE Transactions on Automatic Control, 52(5):943-948, 2007. 
[5] Y.-P. Tian, C.-L. Liu, Consensus of Multi-agent Systems with Diverse Input and Communication Delays, IEEE Transactions on Automatic Control, 53(9):2122-2128, 2008.

[6] W. Wang, J.J.E. Slotine, Contraction Analysis of Time-delayed Communication Delays, IEEE Transactions on Automatic Control, 51(4):712-717, 2006.

[7] Y.-G. Sun, L. Wang, Consensus of Multi-agent Systems in Directed Networks with Nonuniform Time-Varying Delays, IEEE Transactions on Automatic Control, 54(7):1607-1613, 2009.

[8] J. Wang, N. Elia, Consensus over Network with Dynamic Channels, Proc. of the 2008 American Control Conference, Seattle, pp.2637-2642, 2008.

[9] M. Cao, A.S. Morse, B.D.O. Anderson, Reaching an Agreement Using Delayed Information, Proc. of the 45th IEEE Conference on Decision and Control, San Diego, pp.3375-3380, 2006.

[10] F. Xiao, L. Wang, Asynchronous Consensus in Continuous-time Multi-agents with Switching Topology and Time-varying Delays, IEEE Transactions on Automatic Control, 53(8):1804-1816, 2008.

[11] V.D. Blondel, J.M. Hendrickx, A. Olshevsky, J.N. Tsitsiklis, Convergence in Multi-agent Coordination, Consensus, and Flocking, Proc. of the 44th IEEE Conference on Decision and Control, Seville, pp.2996-3000, 2005.

[12] V. Gazi, Stability of an Asynchronous Swarm with Time-dependent Communication Links, IEEE Transactions on Systems, Man, and Cybernetics-Part B: Cybernetics, 38(1):267-274, 2008.

[13] J. Hu, Y. Hong, Leader-following Coordination of Multi-agent Systems with Coupling Time Delays, Physica A, 374(2):853-863, 2007.

[14] H. Su, X. Wang, Second-order Consensus of Multiple Agents with Coupling Delay, Proc. of the 7th world Congress on Intelligent Control and Automation, Chongqing, pp.7181-7186, 2008.

[15] P. Lin, Y. Jia, J. Du, S. Yuan, Distributed Consensus Control for Second-order Agents with Fixed Topology and Time-delay, Proc. of the 26th Chinese Control Conference, Zhangjiajie, pp.577-581, 2007.

[16] W. Yang, A.L. Bertozzi, X. Wang, Stability of a Second Order Consensus Algorithm with Time Delay, Proc. of the 47th IEEE Conference on Decision and Control, Cancun, pp.2926-2931, 2008.

[17] D.J. Lee, M.K. Spong, Agreement with Non-uniform Information Delays, Proc. of the 2006 American Control Conference, Minneapolis, pp.756-761, 2006.

[18] S. Kawamura, M. Svinin (eds.), Advances in Robot Control: From Everyday Physics to HumanLike Movements, Berlin: Springer-Verlag, 2006, pp.107-134.

[19] P. Lin, Y. Jia, Consensus of Second-order Discrete-time Multi-agent Systems with Nonuniform Time-delays and Dynamically Changing Topologyies, Automatica, 45(9):2154-2158, 2009.

[20] Y.-P. Tian, C.-L, Liu, Robust Consensus of Multi-agent Systems with Diverse Input Delays and Asymmetric Interconnection Perturbations, Automatica, 45(5):1374-1353, 2009.

[21] Y.-P. Tian, G. Chen, Stability of The Primal-dual Algorithm for Congestion control, International Journal of Control, 79(6):662-676, 2006.

[22] R. Pedrami, B.W. Gordon, Control and Analysis of Energetic Swarm Systems, Proc. of the 2007 American Control Conference, New York, pp.1894-1899, 2007. 
[23] I. Lestas, G. Vinnicombe, Scalable Robustness for Consensus Protocols with Heterogeneous Dynamics, Proc. of the 16th IFAC World Congress, Prague, 2005.

[24] Z. Lin, B. Francis, M. Maggiore, Necessary and Sufficient Graphical Conditions for Formation Control of Unicycles, IEEE Transactions on Automatic Control, 50(1):121-127, 2005.

[25] C.A. Desoer, Y.T. Wang, On the Generalized Nyquist Stability Criterion, IEEE Transactions on Automatic Control, 25(2):187-196, 1980.

Cheng-Lin Liu was born in China in 1981. He got PHD at Southeast University in 2008. Since 2008, He is a faculty member at Institute of Automation, Jiangnan University, China. His current research interests include Internet congestion control and coordination control of multi-agent systems.

Fei Liu was born in China in 1965. He is a professor at Institute of Automation, Jiangnan University, China. His research interests include the theory and application of advanced process control, process monitoring and diagnose on industrial system, and integrated automatic system for fermentation process. 